\title{
Using Electrochemistry - Total Internal Reflection Imaging Ellipsometry to Monitor Biochemical Oxygen Demand on the Surface Tethered Polyelectrolyte Modified Electrode
}

\author{
Wei Liu ${ }^{a, b}$, Meng Li ${ }^{a, b}$, Bei'er $\mathrm{lv}^{c}$, YanYan Chen ${ }^{c}$, Hongwei Ma ${ }^{c}$ and Gang $\operatorname{Jin}^{a}$ \\ ${ }^{a}$ NML, Institute of Mechanics, Chinese Academy of Sciences, \# 15, Bei-si-huan West Road, \\ Beijing, 100190, China; \\ ${ }^{b}$ University of Chinese Academy of Sciences, \# 19, Yu-quan Road, Beijing, 100049, China; \\ ${ }^{c}$ Suzhou Institute of Nano-Tech and Nano-Bionics, Chinese Academy of Science, Suzhou, \\ 21513, China
}

\begin{abstract}
Our previous work has proposed an electrochemistry - total internal reflection imaging ellipsometry (EC-TIRIE) technique to observe the dissolved oxygen (DO) reduction on Clark electrode since high interface sensitivity makes TIRIE a useful tool to study redox reactions on the electrode surface. To amplify the optical signal noise ratio (OSNR), a surface tethered weak polyelectrolyte, carboxylated poly(oligo(ethylene glycol) methacrylate-random2-hydroxyethylmethacrylate) (abbreviated as carboxylated poly(OEGMA-r-HEMA)), has been introduced on the electrode surface. Since Clark electrode is widely used in biochemical oxygen demand (BOD) detection, we use this technique to measure BOD in the sample. The dynamic range of the system is from $0 \sim 25 \mathrm{mg} / \mathrm{L}$. Two samples have been measured. Compared with the conventional method, the deviation of both optical and electrical signals are less than $10 \%$.
\end{abstract}

Keywords: Total internal reflection imaging ellipsometry, Surface tethered weak polyelectrolyte, Biochemical oxygen demand

\section{INTRODUCTION}

Biochemical oxygen demand is widely used as an indication of the organic pollution of water, which measures the amount of dissolved oxygen (DO) consumed by aerobic biological organism in water to decompose organic material in a given water sample at certain temperature during a specific time period. ${ }^{1}$ Conventionally, we use five-day Biochemical oxygen demand $\left(\mathrm{BOD}_{5}\right)$ to determinate the $\mathrm{BOD}$ value: which gives milligrams of oxygen consumed per litre of the sample after 5 days of incubation at $20^{\circ} \mathrm{C}$. As $\mathrm{BOD}_{5}$ is time-consuming and the procedure is complicated, an alternative for rapid BOD measurement is needed. BOD biosensors have been thus developed for this purpose. As the DO variation is the key to BOD measurement, a typical BOD biosensor is composed of an oxygen electrode and a microbial membrane. When the organic pollutants in the sample is degraded by the microorganism in the membrane, the oxygen electrode detects the DO change by the degradation.

Our previous work has demonstrated TIRIE have the ability to observe the DO reduction on the surface of Clark electrode for it enjoys high interface sensitivity. ${ }^{2}$ However, the low Optical signal noise ratio (OSNR) has limited its application as BOD biosensor. When DO is reduced on the electrode, the local $\mathrm{pH}$ in the vicinity of the electrode surface will change because of the hydroxyl ions reduced from DO. On the other hand, carboxylated poly(OEGMA-r-HEMA)) is found sensitive to $\mathrm{pH}$ of the medium: it will swell when $\mathrm{pH}$ rise from 2 to $7.4,{ }^{3}$ which makes TIRIE an especially useful tool to detect the structure change of the surface tethered weak polyelectrolyte on the sensing surface. Thus, we modify the carboxylated poly(OEGMA-r-HEMA) on the electrode to amplify the OSNR and use the EC-TIRIE system to perform rapid BOD measurement in this paper.

Further author information: (Send correspondence to Gang Jin)

Gang Jin: E-mail: gajin@imech.ac.cn, Telephone: +8610 82544138

Quantitative Phase Imaging, edited by Gabriel Popescu, YongKeun Park, Proc. of SPIE Vol. 9336,

93360C · @ 2015 SPIE · CCC code: 1605-7422/15/\$18 · doi: 10.1117/12.2078486 


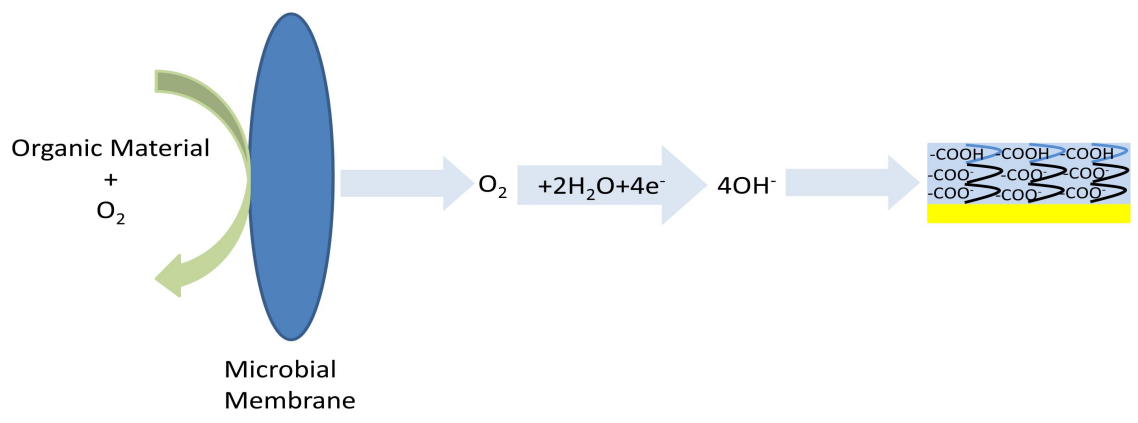

Figure 1: When the organic material in the sample is decomposed by the microbial membrane, the DO concentration of the sample decreases. Since less $\mathrm{DO}$ is reduced to $\mathrm{OH}^{-}$, the local $\mathrm{pH}$ of the electrolyte will decrease the thickness of the polyelectrolyte.

\section{EXPERIMENTAL}

\subsection{Polyelectrolyte Preparation}

For the most widely used BOD biosensor, a microorganism membrane is immobilized on the top of a Clark electrode, which is a typical oxygen electrode. When organic material is consumed by the microbial membrane, DO in the sample will be decreased. At the same time, we polarize the electrode with $-500 \mathrm{mV}$ (vs. $\mathrm{Ag} / \mathrm{AgCl}$ ), hydroxyl ions will be generated by the DO reduction. As the DO concentration of the sample decreases, less hydroxyl ions will be produced. Thus, the local $\mathrm{pH}$ in the neighbourhood of the electrode varies.

Carboxylated poly(OEGMA-r-HEMA) is reported as a surface tethered weak polyelectrolyte sensitive to $\mathrm{pH}$ and ionic strength of the medium. ${ }^{3}$ It will swell from $\mathrm{pH} 2$ to 7.4 : the increase of $\mathrm{pH}$ will make $\mathrm{COOH}$ groups in the structure dissociate as $\mathrm{COO}^{-}$groups. Thus, the electrostatic interactions among $\mathrm{COO}^{-}$groups will stretch the polyelectrolyte. Since TIRIE is sensitive to the interface, we can use EC-TIRIE technique to monitor the swelling behavior of the polyelectrolyte on the sensing surface. In general, the measurement principle is shown as fig. 1. Carboxylated poly(OEGMA-r-HEMA) of $8 \mathrm{~nm}$ were prepared as previously described. ${ }^{3,4}$ Briefly, TIRIE chips (SF10 glass slides covered with a 2-nm-thick Cr adhesive under-layer and a 50-nm-thick gold film) was first modified with initiator thiol and diluent thiol via a self-assembled monolayer (SAM) technique. After the modification, surface initiated polymerization (SIP) was initiated by immersing the SAM modified chips to a reaction solution. By controlling SIP time, the polymer thickness varies. Finally, the polyelectrolyte surfaces were generate by substuting carboxyl groups for hydroxyl groups of poly(OEGMA-r-HEMA).

\subsection{Chemicals and Materials}

PBS buffer (pH 7.4) was purchased from Sigma-Aldrich, hydrochloric acid (HCl) of $37 \%$ concentration from Beijing Chemical Works. All the compounds used in this work were in analytical grade without further purification. Ultrapure water was obtained from a MILLI-Q purification system (18.2 $\mathrm{M} \Omega$ at room temperature) and used to prepare all the solutions.

Since the swelling behavior of the surface tethered weak polyelectrolyte is not only determined by the $\mathrm{pH}$ of the medium but by the ionic strength, the diluted PBS with $\left[\mathrm{Na}^{+}\right]=1.5 \mathrm{mM}$ was optimized as the electrolyte, $\mathrm{pH}$ of which was adjusted to 1 with $\mathrm{HCl}$.

A glucose-glutamic acid (GGA) with $\mathrm{BOD}_{5}$ value $2500 \mathrm{mg} / \mathrm{L}(1705 \mathrm{mg} / \mathrm{L}$ glucose and $1705 \mathrm{mg} / \mathrm{L}$ glutamic acid) is used as BOD standard solution. By diluting the solution with $10 \mathrm{mM}$ PBS, GGA solutions with other $\mathrm{BOD}_{5}$ value are obtained.

The microbial membrane was purchased from NISSIN ELECTRIC. CO. Ltd.. Water samples were taken from lakes in Peking University and Tsinghua University. Before analysis, $\mathrm{pH}$ of both samples was measured. The BOD values of the samples were determined by the conventional $\mathrm{BOD}_{5}$ method. 


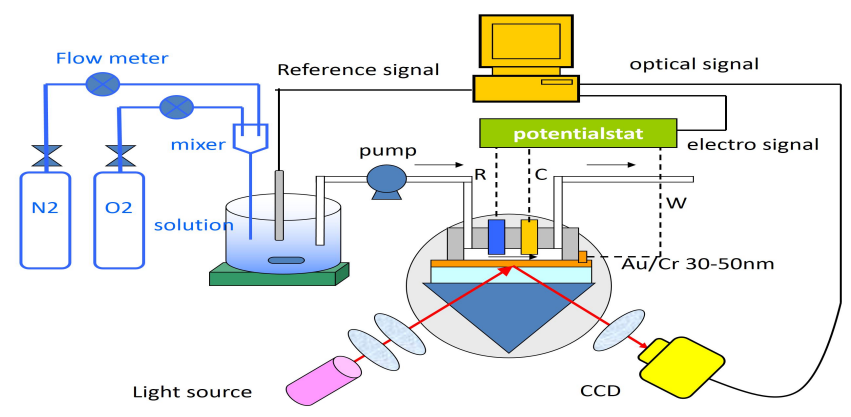

Figure 2: Schematic illustration of the EC-TIRIE system. W represents the working electrode, $\mathrm{R}$ the reference electrode and $\mathrm{C}$ the counter electrode. The sample and the DO saturated electrolyte were dilivered into the experimental cell by a pump. The redox reaction was monitored by both an TIRIE system and an electrochemistry system. An external electrochemistry station was applied for the electrochemical condition. Both the optical and electric signals were recorded by a computer.

\subsection{Measurement Principle and Instrumentation}

High interface sensitivity makes TIRIE a powerful tool to study various physics, chemical and biochemical processes on a substrate surface. For a typical faradic process during electrochemistry, the refractive index near the electrode would be modulated by the redox. ${ }^{2}$ Thus, TIRIE technique can be used to study the redox on the electrode surface.

Figure 2 illustrates the EC-TIRIE setup used for all experiments. The electrochemical experiments are performed using a VersaSTAT 3 electrochemical system (Princeton, U.S.A). A SF10 trapezoidal prism is used with a Xe lamp as the light source and a high-speed CCD camera as the detector. The $632.8 \mathrm{~nm}$ light beam is guided by an optical fiber and expanded by a collimating system. After passing a polarizer and a compensator, the polarized collimated beam propagates perpendicularly to the prism and onto the sensing surface. When the incident angle is optimized as $65^{\circ}$, larger than the critical angle, the evanescent wave appears sharply at the sensing surface to detect the interaction in very shallow depth from the surface. The reflected light carrying the surface information is then imaged by a CCD camera after passing an analyzer. The system works under the null and off-null mode, which almost fulfills the null condition on the sensing surface when no redox reactions occur and detects the thickness change of the surface tethered weak polyelectrolyte under the off-null condition. Combined with an external electrochemical working station, this configuration allowed for simultaneously TIRIE detection of the optical variation on the sensing surfaces of both detection units and the electrochemical measurement. During the measurement, the optical variation of the sensing surface caused by the DO reduction was recorded by TIRIE in grayscale.

\subsection{Experimental Procedure}

To activated the microorganism in the microbial membrane, the membrane needs to be submerge into $10 \mathrm{mM}$ PBS solution at least 72 hours. After the activation, we put the microbial membrane next to the gas permeable membrane. Thus, the electrolyte and the sample solution are separated by the membranes. Oxygen are pumped into the sample to make it DO saturated. For each experiment, a DO saturated PBS buffer are first delivered into the sample compartment of the cell to get the base line of the measurement. Then, a DO saturated sample substitute for the buffer. After the degradation of the sample reaches equilibrium, the DO variation can be calculated from the signal change.

\section{RESULTS AND DISCUSSION}

A typical BOD measurement real-time curve is shown in fig. 3a. To calibrated the EC-TIRIE system, six GGA solutions with known BOD values were used. Both electrical signal and optical signal were linear to the BOD values as shown in fig. 3b. After the calibration, we used the system to measure real water samples and compared with the conventional $\mathrm{BOD}_{5}$ method. The deviation were less than $10 \%$. 


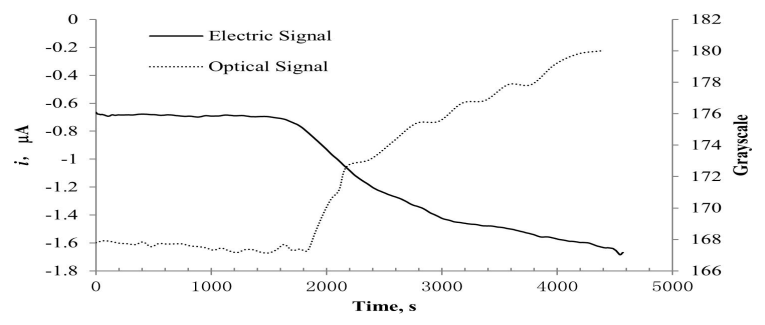

(a)



(b)

Figure 3: 3a: The real-time BOD curve on the carboxlated poly(OEGMA-r-HEMA) covered electrode. During $1400 \mathrm{~s}$, a $25 \mathrm{mg} / \mathrm{L}$ BOD sample has been pumped into the cell; 3b: the BOD calibration curves of EC-TIRE system.

Table 1: The measured BOD value by both conventional $\mathrm{BOD}_{5}$ method and EC-TIRE method. The Sample 1 is from the lake of Peking University and sample 2 from the one of Tsinghua University.

\begin{tabular}{cccccc}
\hline & & \multicolumn{2}{c}{ Optical Signal } & \multicolumn{2}{c}{ Electrical Signal } \\
\cline { 3 - 6 } & $\mathrm{BOD}_{5}(\mathrm{mg} / \mathrm{L})$ & $\mathrm{BOD}_{\mathrm{p}}(\mathrm{mg} / \mathrm{L})$ & Deviation & $\mathrm{BOD}_{\mathrm{c}}(\mathrm{mg} / \mathrm{L})$ & Deviation \\
\hline Sample 1 & 2.8 & 2.570 & $8.2 \%$ & 2.531 & $9.6 \%$ \\
Sample 2 & 2.2 & 1.996 & $9.3 \%$ & 2.122 & $3.5 \%$ \\
\hline
\end{tabular}

\section{CONCLUSIONS}

The surface tethered weak polyelectrolyte films has been introduced on the sensing surface. EC-TIRIE system has been used to observe the BOD of the sample on the modified electrode. Owing to the OSNR amplification of the carboxylated poly(OEGMA-r-HEMA), the dynamic range of the BOD detection has been extended from $0 \sim 25 \mathrm{mg} / \mathrm{L}$. The water samples from Peking University and Tsinghua University have been measured by the system. Compared with the conventional $\mathrm{BOD}_{5}$ method, the deviation of both optical and electrical signals are less than $10 \%$. Thus, EC-TIRIE system enjoys the capability of overcoming the signal shift of conventional rapid BOD measurement. When electric signal drifts, the optical signal can tell whether the electric signal change is caused by different BOD of the sample or other factors. Since TIRIE enjoys the high throughput, the system has the potential to reduce the measurement time further by introducing a reference channel.

\section{ACKNOWLEDGMENTS}

The authors acknowledge the financial support to the National Natupral Science Foundation of China (21305147), to the International Science \& Technology Cooperation Program of China (2012DFG31880), to the National Basic Research Program of China (2015CB352104), to the National High Technology Research Development Program of China (2008AA02Z419), and to the Instrument Developing Project of the Chinese Academy of Sciences (KJCX2-YW-M04, KJCX2-YW-M03).

\section{REFERENCES}

[1] Penn, M. R., Pauer, J. J., and Mihelcic, J. R., "Biochemical oxygen demand," Environmental and ecological chemistry 2, 278 (2009).

[2] Liu, W., Li, M., and Jin, G., "Using electrochemistry - total internal reflection ellipsometry technique to observe the dissolved oxygen reduction on clark electrode," Electrochimica Acta 142(0), 371 - 377 (2014).

[3] Zhang, Y., Lv, B., Lu, Z., He, J., Zhang, S., Chen, H., and Ma, H., "Predicting au-s bond breakage from the swelling behavior of surface tethered polyelectrolytes," Soft Matter 7(24), 11496-11500 (2011).

[4] Zhang, Y., He, J., Zhu, Y., Chen, H., and Ma, H., "Directly observed au-s bond breakage due to swelling of the anchored polyelectrolyte," Chemical Communications 47(4), 1190-1192 (2011). 